\title{
HFPK 334: An unusual Supernova Remnant in the Small Magellanic Cloud
}

\author{
E. J. Crawford and M. D. Filipović \\ University of Western Sydney, Australia \\ e.crawford@uws. edu. au \\ and \\ R. L. McEntaffer, T. Brantseg, K. Heitritter and Q. Roper \\ Department of Physics and Astronomy, University of Iowa, Iowa City, IA 52242 \\ and \\ F. Haberl \\ Max-Planck-Institut für extraterrestrische Physik, Giessenbachstraße, 85748 Garching, Germany \\ and \\ D. Urošević \\ Department of Astronomy, Faculty of Mathematics, University of Belgrade, Studentski trg 16, \\ 11000 Belgrade, Serbia \\ Isaac Newton Institute of Chile, Yugoslavia Branch
}

\begin{abstract}
We present new Australia Telescope Compact Array (ATCA) radio-continuum and XMM-Newton/Chandra X-ray Observatory (CXO) observations of the unusual supernova remnant HFPK 334 in the Small Magellanic Cloud (SMC). The remnant follows a shell type morphology in the radio-continuum and has a size of $\sim 20 \mathrm{pc}$ at the SMC distance. The X-ray morphology is similar, however, we detect a prominent point source close to the center of the SNR exhibiting a spectrum with a best fit powerlaw with a photon index of $\Gamma=2.7 \pm 0.5$. This central point source is most likely a background object and cannot be directly associated with the remnant. The high temperature, nonequilibrium conditions in the diffuse region suggest that this gas has been recently shocked and point toward a younger SNR with an age of $\lesssim 1800$ years. With an average radio spectral index of $\alpha=-0.59 \pm 0.09$ we find that an equipartition magnetic field for the remnant is $\sim 90 \mu \mathrm{G}$, a value typical of younger SNRs in lowdensity environments. Also, we report detection of scattered radio polarisation across the remnant at $20 \mathrm{~cm}$, with a peak fractional polarisation level of $25 \pm 5 \%$.
\end{abstract}


Subject headings: ISM: individual objects (HFPK 334) ISM: supernova remnants pulsars: general X-rays: general

\section{Introduction}

The study of supernova remnants (SNRs) in nearby galaxies is of major interest in order to understand the multi-frequency output of more distant galaxies, and to understand the processes that proceed on local interstellar scales within our own Galaxy. Unfortunately, the distances to many Galactic remnants are uncertain by a factor of $\sim 2$ (eg. Johanson \& Kerton 2009; Green $2009 \mathrm{a}$ b), leading to a factor of $\sim 4$ uncertainty in luminosity and of $\sim 5.5$ in the calculated energy release of the initiating supernova $(\mathrm{SN})$. At an assumed distance of $\sim 60 \mathrm{kpc}$ (Hilditch et al. 2005), the Small Magellanic Cloud (SMC) is one of the prime targets for the astrophysical research of objects, including SNRs. These remnants are located at a known distance, yet close enough to allow a detailed analysis.

SNRs reflect a major process in the elemental enrichment of the interstellar medium (ISM). Multiple supernova explosions over space-time generate super-bubbles typically hundreds of parsecs in extent. Both are among the prime drivers controlling the morphology and the evolution of the ISM. Pulsar wind nebulae (PWN) offer further information, as the SNR shell and PWN together provide unique constraints and insights into the ISM. Their properties are therefore crucial to the full understanding of the galactic matter cycle.

Today, a total number of 24 classified SNRs are known in the SMC (Haberl et al. 2012a c; Filipović et al. 2008; van der Heyden et al. 2004, and references therein). This represents the most complete sample of SNRs in any galaxy. There is one confirmed PWN in the SMC, IKT 16 (Owen et al.2011), and at least three other candidate PWNs, HFPK 334, DEM S5 and IKT 4. The other 20 SNRs range from the very young 1E0102 at 1400 years old to the very old HFPK 419 at 50000 years old (Haberl et al. 2012a), giving an unparalleled insight into the evolution of of SNRs and their environment.

Kahabka et al. (1999) first detected HFPK 334 with Röntgensatellit (ROSAT), and list it as source 179 in their catalogue. Haberl et al. (2000) used advanced data processing and additional available data to extend the Kahabka et al. (1999) catalogue and noted that HFPK 334 was extended at $13 \mathrm{~cm}$. It was Filipović et al. (2008) that provided the first conformation that HFPK 334 was an SNR, albeit an unusual SNR with detectable radio and X-ray emission, but no optical emission (Payne et al. 2007). They also noted a possible central source, leading them to suggest that it may be a PWN, possibly the first (at the time) detected in the SMC. Here, we present new follow-up radio-continuum observations with the Australia Telescope Compact Array (ATCA). This is in addition to previous higher frequency study of Filipović et al. (2008). We also present new Chandra X-ray observations, together with archival XMM- 
Newton observations of the SMC SNR HFPK 334. Therefore we present a new insight on HFPK 334 and clarify the nature of the central point source suggested in previous studies (Filipović et al. 2008).

\section{Observations}

\subsection{The ATCA radio-continuum observations and data reduction.}

We observed HFPK 334 with the ATCA on 2009 January 5 using the 6C array, and on 2009 February 4 using the EW352 array at wavelengths of 20 and $13 \mathrm{~cm}(\nu=1384$ and $2367 \mathrm{MHz})$. The observations were done in an interleaved mode, totaling $\sim 4$ hours of integration over a 12hr period. Source 1934-638 was used for primary calibration and source 0252-712 was used for secondary calibration. The MIRIAD (Sault et al. 1995) and KARMA (Gooch 1996) software packages were used for data reduction and analysis. Images were formed using MIRIAD's multi-frequency synthesis algorithm (Sault \& Wieringa 1994) and natural weighting. They were deconvolved with primary beam correction applied. The same procedure was also used for both $Q$ and $U$ Stokes parameter maps. The mean fractional polarisation at $20 \mathrm{~cm}$ was calculated using flux density and polarisation:

$$
P=\frac{\sqrt{S_{Q}^{2}+S_{U}^{2}}}{S_{I}} \cdot 100 \%
$$

where $S_{Q}, S_{U}$ and $S_{I}$ are integrated intensities for $Q, U$ and $I$ Stokes parameters.

The $20 \mathrm{~cm}$ image (Figure 1) has a resolution of $10^{\prime \prime}$ and an r.m.s. noise of $0.2 \mathrm{mJy} /$ beam. A matched $13 \mathrm{~cm}$ image was produced, with an r.m.s. noise of $0.3 \mathrm{mJy} / \mathrm{beam}$ and used in the calculation of the spectral index.

\section{2. $X M M-N e w t o n$ observations and data reduction}

HFPK 334 was serendipitously observed during an XMM-Newton observation of a candidate supersoft source in the direction of the SMC. The observation (Obs. Id. 0402000101) was performed on 2006 October 3 and further details about instrument setups and data quality can be found in Haberl \& Pietsch (2008). Using data from this observation, Filipović et al. (2008) classified the source as a new candidate SNR (plerionic) and presented images (their Fig. 5) and X-ray spectra obtained by the European Photon Imaging Camera (EPIC). However, their spectral analysis of the SNR spectra did not consider the contribution of a point source near the centre of the remnant, which was revealed in follow-up Chandra observations with superior spatial resolution (see \$2.3 below).

Therefore, we re-extracted the EPIC-pn (Strüder et al. 2001) spectra using the XMM-Newton Science Analysis System (SAS) version 11.0.0. To obtain the best statistics we selected single- and 


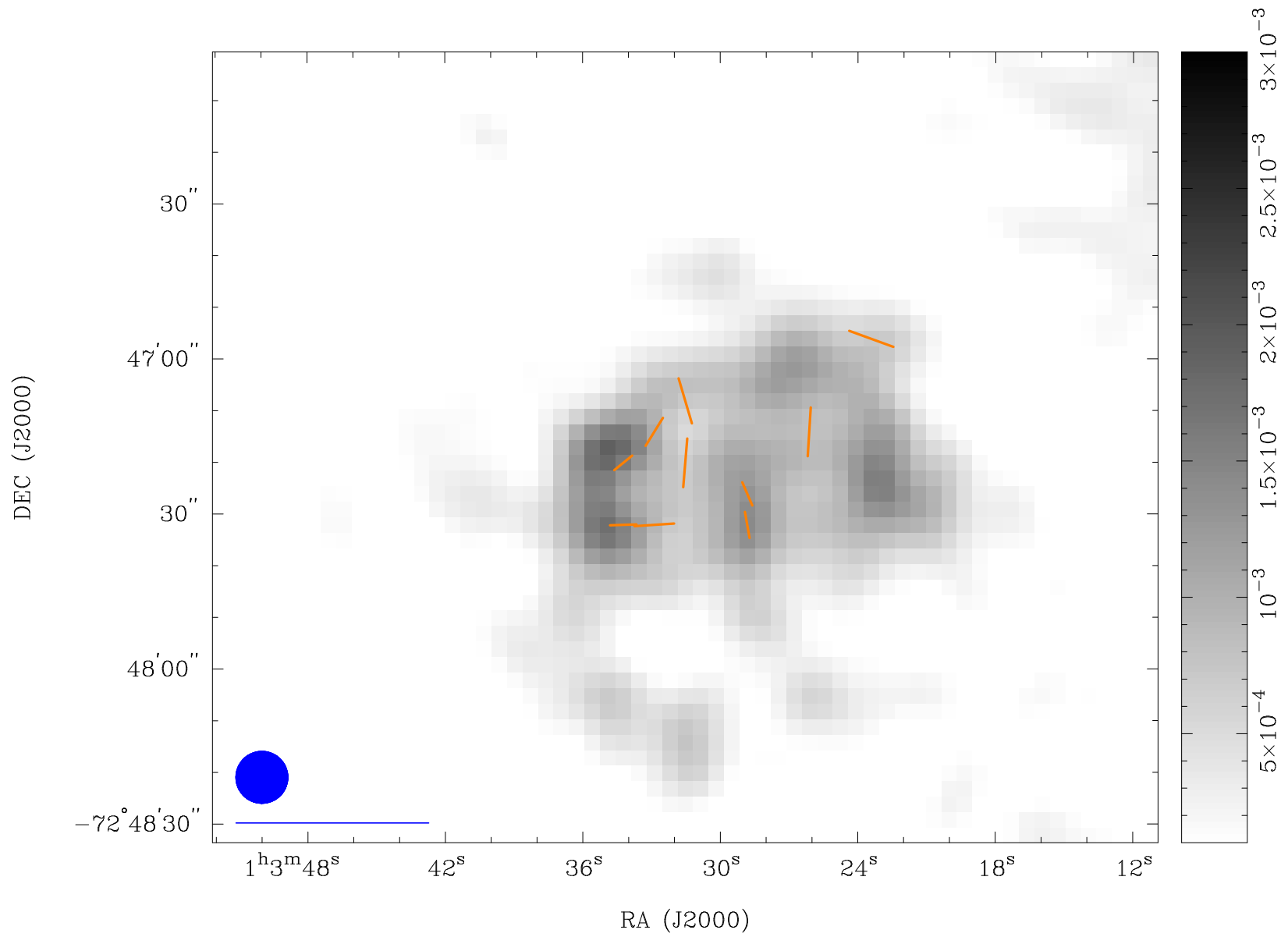

Fig. 1. - ATCA observations of HFPK 334 at $20 \mathrm{~cm}(1384 \mathrm{MHz})$ overlaid with fractional polarised intensity. The ellipse in the lower left corner represents the synthesised beam of $10^{\prime \prime}$ and the line below the ellipse is a polarisation vector of $100 \%$. The peak polarisation is estimated to $\sim 25 \pm 5 \%$. The sidebar quantifies the pixel map in units of Jy/beam. 
double-pixel events with quality flag 0 and binned the source spectrum to a minimum of 20 counts per bin. Source counts were extracted from an ellipse with a size of $40^{\prime \prime} \times 30^{\prime \prime}$, while the background was selected from a nearby source-free circular region with $60^{\prime \prime}$ radius. The net exposure time was $17.5 \mathrm{ks}$.

\subsection{The Chandra X-ray observations and data reduction}

Dedicated X-ray observations of HFPK 334 were carried out with the Chandra X-ray observatory on 2010 December 9 (Obs. Id. 11821) as part of the guaranteed time program available at MPE. The observation was made using ACIS-S and totalled $28.55 \mathrm{ksec}$. The level 1 data were reprocessed to level 2 with standard processing procedures in the Chandra Interactive Analysis of Observations (CIAO, v 4.4; Fruscione et al. 2006) software package with current calibration data from the Chandra Calibration Database (CALDB, v.4.4.2). Good time intervals, charge transfer inefficiency, and time-dependent gain variations were accounted for. The extracted spectra were background-corrected using adjacent regions of the chip that were devoid of emission. The effective exposure time after removing bad events is $28.19 \mathrm{ksec}$.

The raw data are shown in Figure 2, The image has been binned to $\sim 2^{\prime \prime}$ pixels and Gaussiansmoothed over a 3-pixel kernel. The scale ranges from 0.2-90 counts/bin. Due to the low average count rate of $0.017 \mathrm{cts} / \mathrm{sec}$, we treat the diffuse emission as a single region. The red, elliptical region on the right side of Figure 2 encompasses a point source near the center of the remnant. This region is determined using wavdetect in CIAO (Freeman et al. 2002). This tool finds sources in the data set by correlating the image with "Mexican Hat" wavelet functions. The tool then draws an elliptical region around the detected source out to a specified number of standard deviations. In the present case, the detection scales used are 1 and 2 pixels, and the red region size in Figure 2 is $3 \sigma$ where $\sigma$ is the uncertainty in the intensity distribution of the detected point source given the point spread function (PSF) of Chandra. We use the CIAO tool mkpsfmap to determine the expected Chandra PSF at the location of the point source. The extraction region we use for spectral analysis is larger to ensure all point source photons are included. We use an elliptical region centered on the source, at $01^{\mathrm{h}} 03^{\mathrm{m}} 28^{\mathrm{s}} 896-72^{\circ} 47^{\prime} 28.35^{\prime \prime}$, and twice the size of the $3 \sigma$ error contour with axes of $4.7 \times 4.3$ pixels or $2.3^{\prime \prime} \times 2.1^{\prime \prime}$. The remainder of the emission from HFPK 334 exists in a diffuse nebula surrounding the point source. The ellipse labelled "Source" in Figure 2 is the extraction region used for this diffuse emission, with axes of $42^{\prime \prime} \times 49^{\prime \prime}$. The $6 \sigma$ error ellipse used as the extraction region for the point source has been excluded from these data prior to fitting.

Spectra for each of these regions are extracted using the specextract tool in CIAO. This tool automatically creates ancillary response files and redistribution matrix files for each region. A background for the diffuse emission is created from the two circular regions shown on the exterior of the remnant. For the point source the background used is an elliptical annulus surrounding the extraction region with the intent of subtracting any diffuse emission that may be confusing the 


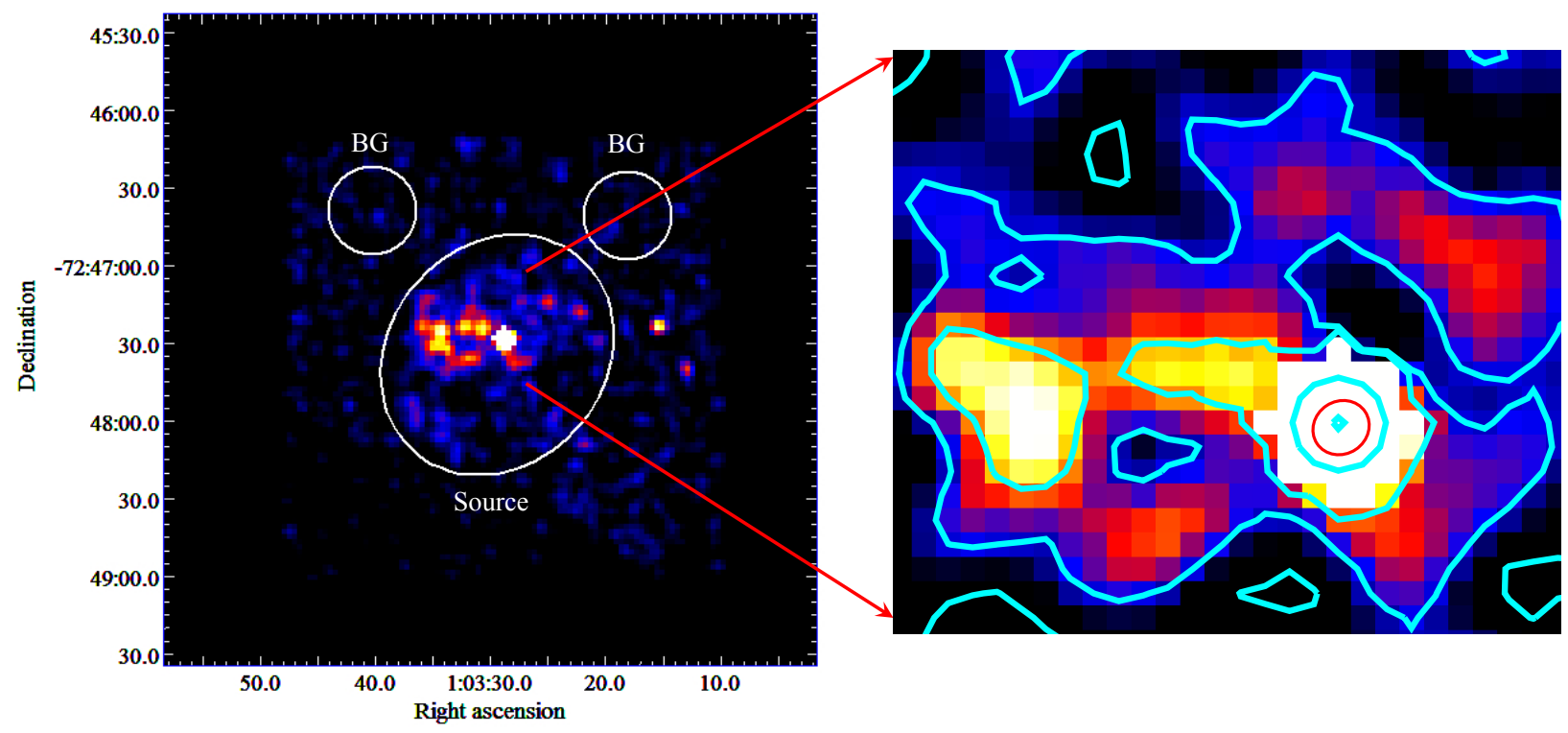

Fig. 2.- Left-Image of the raw data from the Chandra ACIS S2 CCD. The data have been binned to $2^{\prime \prime}$ pixels and Gaussian-smoothed with a 3-pixel kernel radius to a final scale of 0.2-90 counts/pixel. The white ellipsoidal region labeled "Source" is used for spectral analysis with regions labeled "BG" serving as background subtraction regions. Right-A close-up of the central emission using the same binning and smoothing parameters. Cyan contours outline the emission at $1.0,1.8,2.5,20$, and 40 counts per pixel. The red ellipse is the 6 sigma error ellipse for the point source position, as detected using the CIAO tool wavdetect. 
source spectrum. The outer bound of this background annulus measures $11.5 \times 10.6$ pixels $(15 \sigma)$ with an inner bound consistent with the extraction region. The spectral resolution obtained by Chandra is $\sim 5-20(E / \Delta E)$ over the energy band used, 0.5-8.0 keV. Data below $0.5 \mathrm{keV}$ and above $8.0 \mathrm{keV}$ are not used, owing to a combination of uncertain ACIS calibration and lack of flux. For the diffuse emission, data above $2.0 \mathrm{keV}$ is consistent with zero in the Chandra data. The data are binned to at least 20 counts per bin to allow the use of Gaussian statistics.

\section{X-Ray Spectral Fitting}

To analyze the extracted spectra, we use the X-ray spectral fitting software, XSPEC (Arnaud 1996), and the CIAO modelling and fitting package, Sherpa (Freeman et al. 2001).

\subsection{Chandra}

The superior spatial resolution of Chandra allows us to separate the X-ray spectra of the point source and the extended emission from the SNR. For the point source extraction we attempt a variety of fits that examine a range of possibilities for the nature of the emission. These include a powerlaw model to see if the emission is consistent with a pulsar wind nebula (PWN), neutron star, or background AGN, and blackbody and neutron star atmosphere models to test for the presence of a compact central object (CCO). We apply these models to the Chandra data since we cannot extract a point source separately from the diffuse emission in the $X M M-N e w t o n$ data.

In the diffuse region, if the emission is caused by shock-heating of the ejecta or interstellar medium (ISM), we expect to see prominent emission lines. Depending on the plasma density and the time since it has been shocked, we expect the emitting material to be in either collisional ionization equilibrium (CIE; if the plasma has high density or was shocked a long time ago) or in nonequilibrium ionization (NEI). We use the xsvnei model for nonequilibrium conditions (Borkowski et al. 1994, 2001; Hamilton et al. 1983, Liedahl et al. 1995). This model simulates a singletemperature plasma uniformly shocked at a specific time in the past. Although this assumption is very simplified, as SNR plasmas contain a wide range of temperatures and ionization timescales, our data is of insufficient quality to provide meaningful constraints on the more physically realistic xsvpshock or xsvedov models, which account for the range of temperatures and ionization timescales produced by the passage of a plane-parallel (xsvpshock) or spherical (xsvsedov) model. We therefore treat the plasma temperature and ionization timescale obtained with this model as average values. We replace the default xsvnei line list with an augmented list developed by Kazik Borkowski

that includes more inner shell processes especially for the Fe-L lines (Badenes et al. 2006). For equilibrium conditions we use the xsvapec model, which uses an updated version of the ATOMDB 
code (v2.0.1; Smith et al. 2001; Foster et al. 2011) to model the emission spectrum. We include a second temperature component to these fits if it is determined statistically relevant as inferred from an F-test (probabilites $<0.05$ indicate a statistical improvement given the additional component). In addition, we investigate the significance of a contribution from a non-thermal component by including the xssrcut and/or xspowerlaw models in the diffuse region (Reynolds \& Keohane 1999. Reynolds 1998).

To account for interstellar absorption along the line of sight, the above models are convolved with two photoelectric absorption models (xsphabs), one of which is held at our Galactic column density along this line of sight, $N_{H}=2.8 \times 10^{20} \mathrm{~cm}^{-2}$ (a high resolution foreground H I map was kindly provided by Erik Müller, see also Müller et al. (2003)), with solar abundances, and the other allowed to vary to account for absorption in the SMC at SMC abundances. The SMC abundances are taken from Russell \& Dopita (1992) and relative to solar are: He 0.83, C 0.13, N 0.05, O 0.15, $\mathrm{Ne} 0.19, \mathrm{Mg} 0.24$, Si 0.28, S 0.21, Ar 0.16, Ca 0.21, Fe 0.20, and Ni 0.40. Emission line lists in the 0.5-2.0 keV energy range for plasmas with temperatures $k T \sim 0.09-2.0 \mathrm{keV}$ show that the emission is dominated by highly ionized states of $\mathrm{C}, \mathrm{N}, \mathrm{O}, \mathrm{Ne}$, and Fe with contributions from $\mathrm{Mg}$ and Si. The spectral fits begin with all abundances frozen to SMC levels. A given element is allowed to vary if it significantly improves the fit. Dielectronic recombination rates are taken from Mazzotta et al. (1998) with solar abundances from Wilms et al. (2000) and cross-sections from Balucinska-Church \& McCammon (1992).

\subsection{Simultaneous Chandra/XMM-Newton analysis}

To better constrain our fit parameters we perform simultaneous fitting of Chandra ACIS and $X M M$-Newton EPIC-pn data in Xspec. The spatial resolution of XMM-Newton is insufficient to extract the point source and diffuse emission separately. However, the additional data may further constrain the physical properties of the source. Also, flux variations between the different epochs of the Chandra and XMM-Newton data may indicate variability in the central source. The fit data consist of three inputs: the point source extraction from Chandra, the diffuse emission extraction from Chandra, and the entire source in the XMM-Newton data. The parent fit model is the same as that found for the Chandra data, a non-equilibrium ionization model for the diffuse component with an additional powerlaw component for the point source. Each component is allowed a separate photoelectric absorbing column in the LMC with a global, galactic absorption also applied with a frozen value of $N_{H}=2.8 \times 10^{20} \mathrm{~cm}^{-2}$, as described above [phabs $\times($ phabs $\times$ vnei + phabs $\times$ powerlaw $\left.)\right]$. The three data sets are fit simultaneously with the parent model using the following method: the Chandra point source is fit with the thermal model normalization set to 0; the Chandra diffuse source is fit with the powerlaw norm set to 0; the XMM-Newton data norms for both components are allowed to vary; all thermal models have their abundances, absorbing column, ionization parameter, temperature, and normalization linked; all powerlaw models have their absorbing column, and photon index linked; the Chandra point source powerlaw norm is frozen to its best fit value while the 
$X M M$-Newton powerlaw norm is thawed to investigate variability. We performed various iterations of freezing/thawing various parameters to find the tightest parameter constraints, e.g. thawing the Chandra point source powerlaw norm, but find no additional limitations. Furthermore, these additional fit parameters do not result in a lower reduced $\chi^{2}$. The final fit with the best statistic and parameter constraints is shown in Table 1.

\section{Discussion}

\subsection{Radio}

HFPK 334 has a clumpy appearance, with a knot of emission at the centre, which lead Filipović et al. (2008) to suggest that it was likely to contain a PWN, centered at $01^{\mathrm{h}} 03^{\mathrm{m}} 29.5-72^{\circ} 47^{\prime} 20^{\prime \prime}$ with the enclosing remnants extent of $70^{\prime \prime} \times 40^{\prime \prime}(20 \times 12 \mathrm{pc})$ at $\mathrm{PA}=-70^{\circ}$. Using the flux density measurements and images of Filipović et al. (2008) along with our new measurements at $20 \mathrm{~cm}$ and $13 \mathrm{~cm}$ of $26.9 \pm 1.3 \mathrm{mJy}$ and $18.9 \pm 1.5 \mathrm{mJy}$ respectively, we estimate a spectral index $\alpha=-0.59 \pm 0.09$ where $S_{\nu} \propto \nu^{\alpha}$. This estimate includes emission from the entire remnant and central object.

We also created an image from just the longest ATCA baselines (i.e. those to ATCA antenna 6 ), which shows no indication of a central point source, to a $3 \sigma$ detection limit of $\sim 0.3 \mathrm{mJy}$. However, the extended radio emission aligns well with the diffuse X-ray emission (Figure 3 ).

Linear polarisation images were also formed, as shown in Figure 1. The polarised emission is unordered, with a maximum of $25 \pm 5 \%$, and indicates a random magnetic field. We note that this order of polarisation from HFPK 334 is relatively high when compared to other SNRs in the MCs for which the typical range is $\mathbf{0 - 2 0 \%}$ (Crawford et al. $2008 \mathrm{a} \mid \mathbf{b}, \mathbf{2 0 1 0}$; Cajko et al. 2009 ; Bozzetto et al. 2010, 2012a b c d, 2013, 2014a, b; Grondin et al. 2012; De Horta et al. 2012, 2014; Haberl et al. 2012b; Maggi et al. 2012; Kavanagh et al. 2013).

With a radio surface brightness of $0.36 \times 10^{-20} \mathrm{~W} \mathrm{~m}^{-2} \mathrm{~Hz}^{-1} \mathrm{sr}^{-1}$, and a diameter of $\sim 20 \mathrm{pc}$, the position of HFPK 334 on the surface brightness-diameter diagram of Berezhko \& Völk (2004, their Figure 6), leads us to infer an explosion energy to be in the order of $2 \times 10^{51}$ ergs. We calculate an equipartition magnetic field of $\sim 90 \mu \mathrm{G}$ (Arbutina et al. 2012) which is high for an MC SNR (Bozzetto et al. in prep). Assuming a strong shock passing through the ISM one can expect magnetic field of up to $\sim 20 \mu \mathrm{G}$ (the SMC magnetic field is $\sim 3 \mu \mathrm{G}$ Mao et al. (2008)). Another mechanism, so called amplification of the magnetic field (added to simple compression by the shock) is therefore necessary to explain such a high magnetic field of $90 \mu \mathrm{G}$. The amplification of magnetic field is process driven by very fast shocks of young SNRs. Because of this, a spectral index of -0.59 , the location in the surface brightness-diameter diagram, and moderate amplification (strong amplification would lead to a field of a few hundred $\mu \mathrm{G}$ (Telezhinsky et al. 2012)) of magnetic field suggesting that this SNR is on somewhat younger age i.e. 
Table 1. Best fit parameters for the spectra from different extraction regions

\begin{tabular}{lccccccccc}
\hline \hline \multicolumn{1}{c}{ Region } & $\chi^{2} /$ dof & $\begin{array}{c}N_{H, P L} \\
\left(10^{22} \mathrm{~cm}^{-2}\right)\end{array}$ & $\begin{array}{c}N_{H, \text { vnei }} \\
\left(10^{22} \mathrm{~cm}^{-2}\right)\end{array}$ & $\mathrm{b}$ & $\begin{array}{c}k T \\
(\mathrm{keV})\end{array}$ & $\begin{array}{c}\tau \\
\left(10^{9} \mathrm{~cm}^{-3} \mathrm{~s}\right)\end{array}$ & $\begin{array}{c}\text { norm } \\
10^{-5} A^{\mathrm{d}}\end{array}$ & $\begin{array}{c}\text { norm } \\
10^{-5} B^{\mathrm{e}}\end{array}$ \\
\hline Point source & $13 / 19$ & $0.8 \pm 0.4$ & $\ldots$ & $2.7 \pm 0.5$ & $\ldots$ & $\ldots$ & $5 \pm 2$ & $\ldots$ \\
Diffuse emission & $18.0 / 19$ & $\ldots$ & $<0.5$ & $\ldots$ & $1_{-1}^{+3}$ & $6 \pm 3$ & $\ldots$ & $5_{-2}^{+21}$ \\
Combined $^{\mathrm{f}}$ & $84 / 89$ & $0.8 \pm 0.1$ & $0.1_{-0.1}^{+0.3}$ & $2.8 \pm 0.2$ & $1.3_{-0.8}^{+1}$ & $7_{-2}^{+5}$ & $8 \pm 1^{\mathrm{g}}$ & $6_{-2}^{+0.2}$ \\
\hline
\end{tabular}

a Absorption column in the SMC along the line of sight to the point source.

${ }^{\mathrm{b}}$ Absorption column in the SMC along the line of sight to the diffuse emission.

${ }^{\mathrm{c}}$ Ionization timescale, defined as $n_{e} t$, where $t$ is the time since the plasma was shocked.

dNormalization parameter. $A=1$ photon $\mathrm{keV}^{-1} \mathrm{~cm}^{-2} \mathrm{~s}^{-1}$ at $1 \mathrm{keV}$.

enormalization parameter. $B=\left[10^{-14} /\left(4 \pi D^{2}\right)\right] \int n_{e} n_{H} d V$, where $D$ is the distance to the SMC $(60 \mathrm{kpc})$ anc the integral is the volume emission measure.

${ }^{\mathrm{f}}$ This is a parallel fit with XMM-Newton, Chandra point source, and Chandra diffuse emission data sets.

'Normalization parameter for the XMM-Newton data. The Chandra data are set to the best fit normalization.

Note. - Quoted errors are 90\% confidence intervals for the parameter in question. 


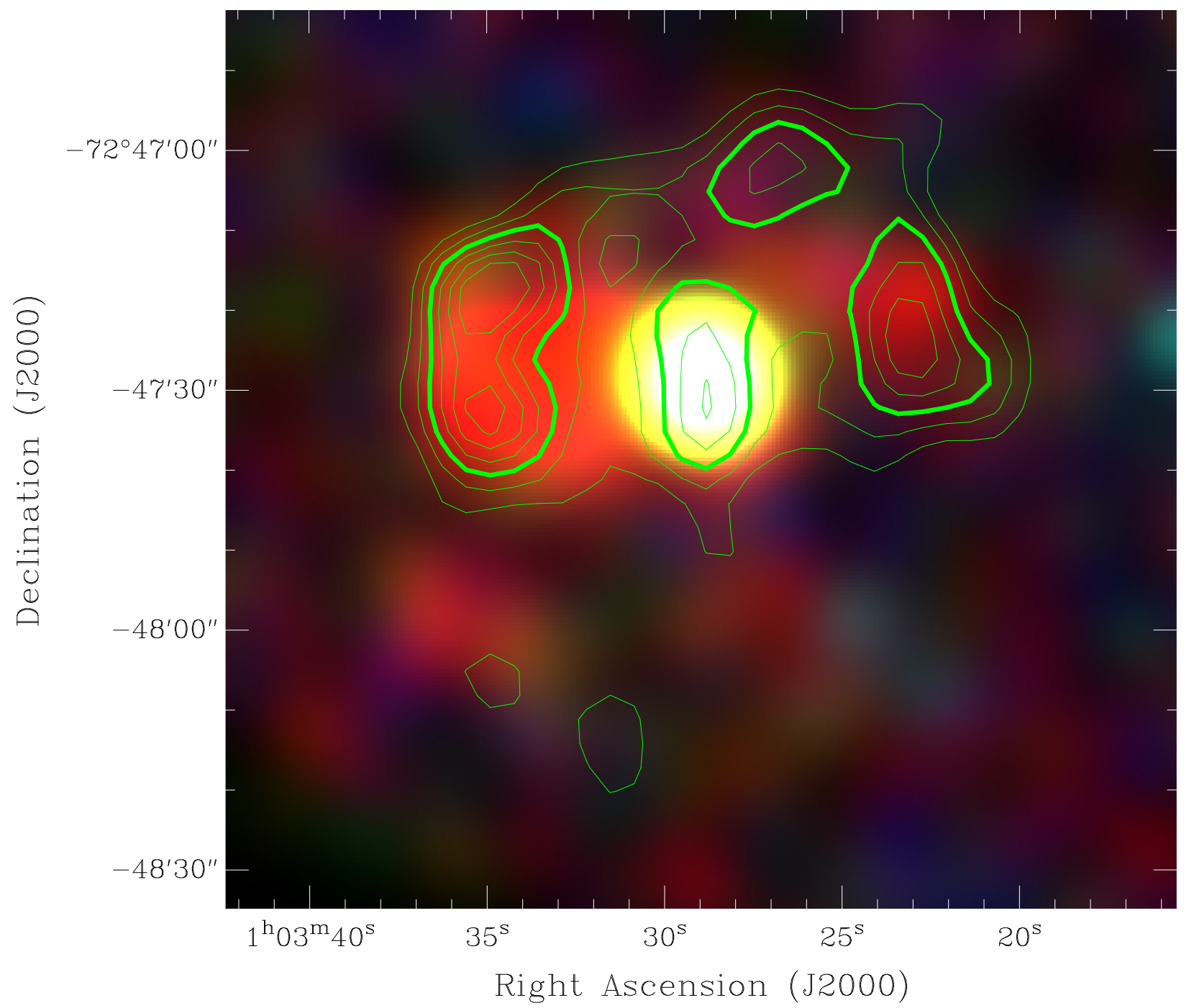

Fig. 3.- Chandra three colour composite (red: 0.3-1.0 keV (soft), green: 1.0-2.0 keV (medium), blue: $2.0-6.0 \mathrm{keV}$ (hard)) smoothed with a Gaussian width to match $20 \mathrm{~cm}$ radio image. The radio contours are $0.6-1.6 \mathrm{mJy} /$ beam in $0.2 \mathrm{mJy} /$ beam steps. 
$<5000$ yr.

\subsection{X-Ray}

The best fit parameters for the two Chandra regions are given in Table 1. The point source region is best fit by a powerlaw with photon index $\Gamma=2.7 \pm 0.5$. The fit is shown overlaid on the data in Figure 4. Attempts at fitting the point source spectrum with other possible models, such as blackbody and neutron star atmosphere to test for a leftover compact object, were generally reasonable but the absorbing column is poorly constrained. A neutron star atmosphere model, $n s a$ in Xspec, resulted in a fit statistic of $\chi^{2} / d o f=15.3 / 19$ and an effective temperature of $\log T=6.5$, but the absorbing column was low, with an upper limit of $3 \times 10^{20} \mathrm{~cm}^{-2}$.

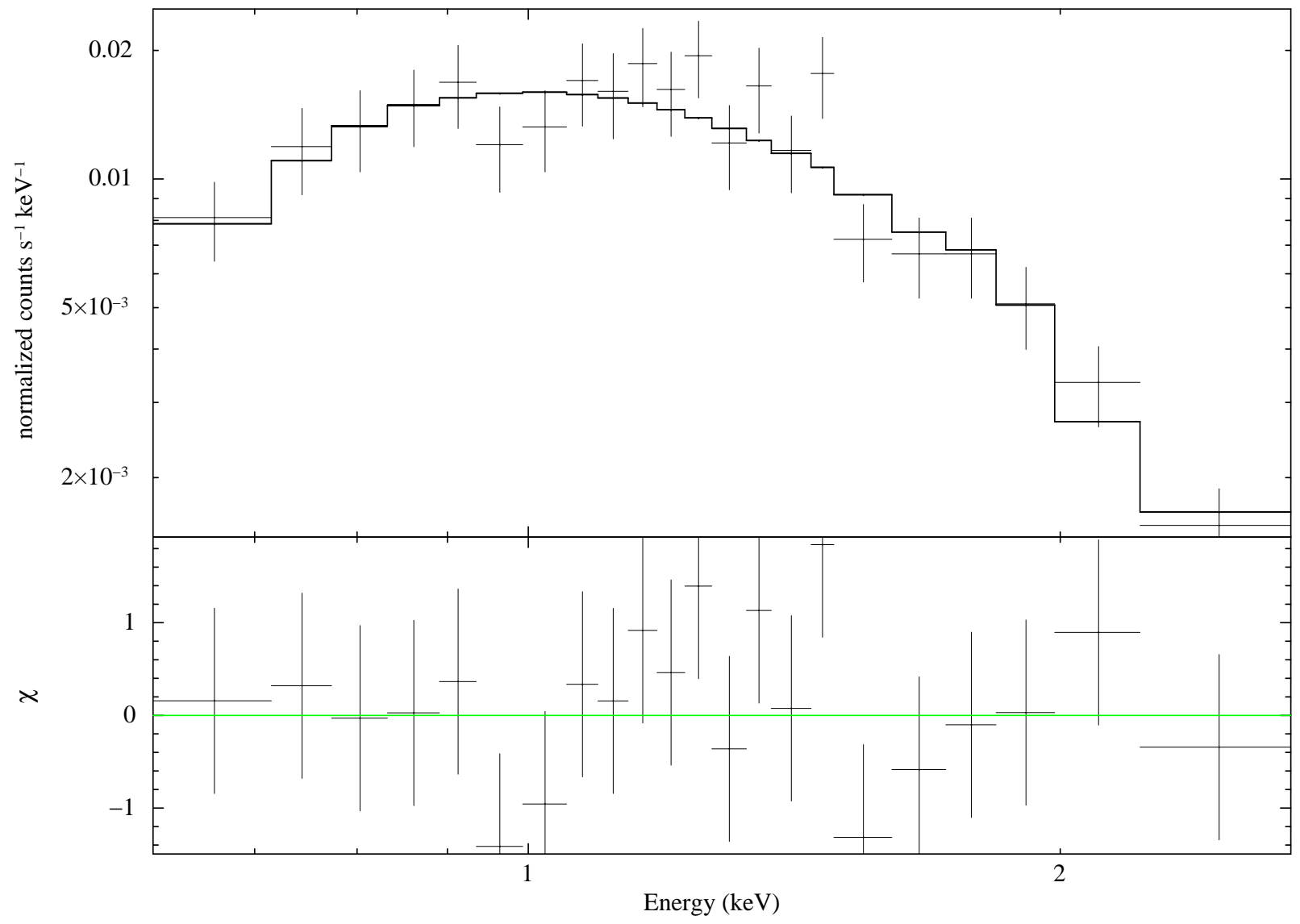

Fig. 4.- Chandra spectrum extracted from the point source region with the best fit powerlaw model overlaid.

We also model the spectrum using the bbody model resulting in a fit statistic of $\chi^{2} / d o f=$ 17.5/19 and temperature of $k T \sim 0.4 \mathrm{keV}$. Again, the absorbing column is ill-constrained, with 
an upper limit of $1.6 \times 10^{21} \mathrm{~cm}^{-2}$. Using the energy band common to both Chandra and XMMNewton, $0.5-4.0 \mathrm{keV}$, we can calculate the fluxes and luminosities for these models. The flux values are consistent and range from $7.5 \times 10^{-14} \mathrm{erg} \mathrm{cm}^{-2} \mathrm{~s}^{-1}$ to $8.7 \times 10^{-14} \mathrm{erg} \mathrm{cm}^{-2} \mathrm{~s}^{-1}$ for the various models resulting in luminosities from $3.3 \times 10^{34} \mathrm{erg} \mathrm{s}^{-1}$ to $3.8 \times 10^{34} \mathrm{erg} \mathrm{s}^{-1}$ assuming a $60 \mathrm{kpc}$ distance to the object.

The X-ray position of the central point source is $01^{\mathrm{h}} 03^{\mathrm{m}} 28^{\mathrm{s}} 896-72^{\circ} 47^{\prime} 28.35^{\prime \prime}$. We find a faint $\left(\mathrm{I}_{m a g}=20.849\right.$ and $\left.\mathrm{V}_{m a g}=21.628\right)$ object in OGLE survey (Udalski et al. 1998) at a distance of $\sim 1.52^{\prime \prime}$. As the $2 \sigma$ error of our X-ray positional estimate is in order of $1.4^{\prime \prime}$ and OGLE positional error is $\sim 0.2^{\prime \prime}$ we claim that this is fully consistent with an association (according to the cumulative Rayleigh distribution the probability for the counterpart to be found within $1.5^{\prime \prime}$ is $88 \%$ ). We calculate the optical to X-ray flux ratio to be 0.91 , indicating background object, a foreground star would have a ratio less than -1 and a foreground neutron star would have a ratio greater than 4 (Maccacaro et al. 1988; Haberl 2004; Sturm et al. 2013).

The diffuse emission region cannot be described by a powerlaw and is best fit with a nonequilibrium thermal plasma at a temperature of $k T=1_{-1}^{+3} \mathrm{keV}$. This fit is shown in Figure 5 . The fit could not be improved with variable abundance of any element or combinations thereof.

The results of the combined fit are also given in Table 1. In addition to the powerlaw component for the point source, a nonequilibrium plasma is again found as the best explanation for the diffuse emission. This thermal component has a temperature comparable to that found when considering Chandra data alone. The additional XMM-Newton data allow for tighter constraints on the temperature and ionization parameter for the thermal plasma, and normalizations for both components. However, the combined fit is still not improved by thawing any elemental abundances. The best fits to these data are shown in Figure 6 .

The spectral index of the high energy emission, $\alpha=2.7 \pm 0.5$, is high, but given the large error bars, barely consistent with the range expected if the emission was arising from a PWN. Given a lack of plerionic remnants in the SMC we can use the LMC for comparison. PWN containing remnants in the LMC typically have a lower index: $\alpha=1.0 \pm 0.2$ in SNR 0453-68.5 (McEntaffer et al. 2012); $\alpha=0.4-1.4$ for a series of annular regions encompassing the PWN of SNR 0540-69.3 (Petre et al. 2007); $\alpha=1.2 \pm 0.3$ in N 206 (SNR 0532-71.0) (Williams et al. 2005); $\alpha=0.57_{-0.06}^{+0.05}$ for DEM L241 (Bamba et al. 2006); and $\alpha \sim 1.2-1.6$ over several regions from N 157B (Chen et al. 2006). At SMC distance the calculated luminosity would be consistent with that expected expected from a PWN. Even though a PWN fits in nicely with the picture of a young SNR and the powerlaw fit was statistically best, the photon index is more compatible with an AGN interpretation, which typically have typical $\Gamma \sim 1.5-2.1$ (Ishibashi \& Courvoisier 2010).

As shown in Table 1, the thermal and powerlaw components of the combined fits have different column densities. We expect that if the point source were associated with the surrounding SNR, the two components would have consistent absorbing columns. The significantly higher column density of the point source implies that it 


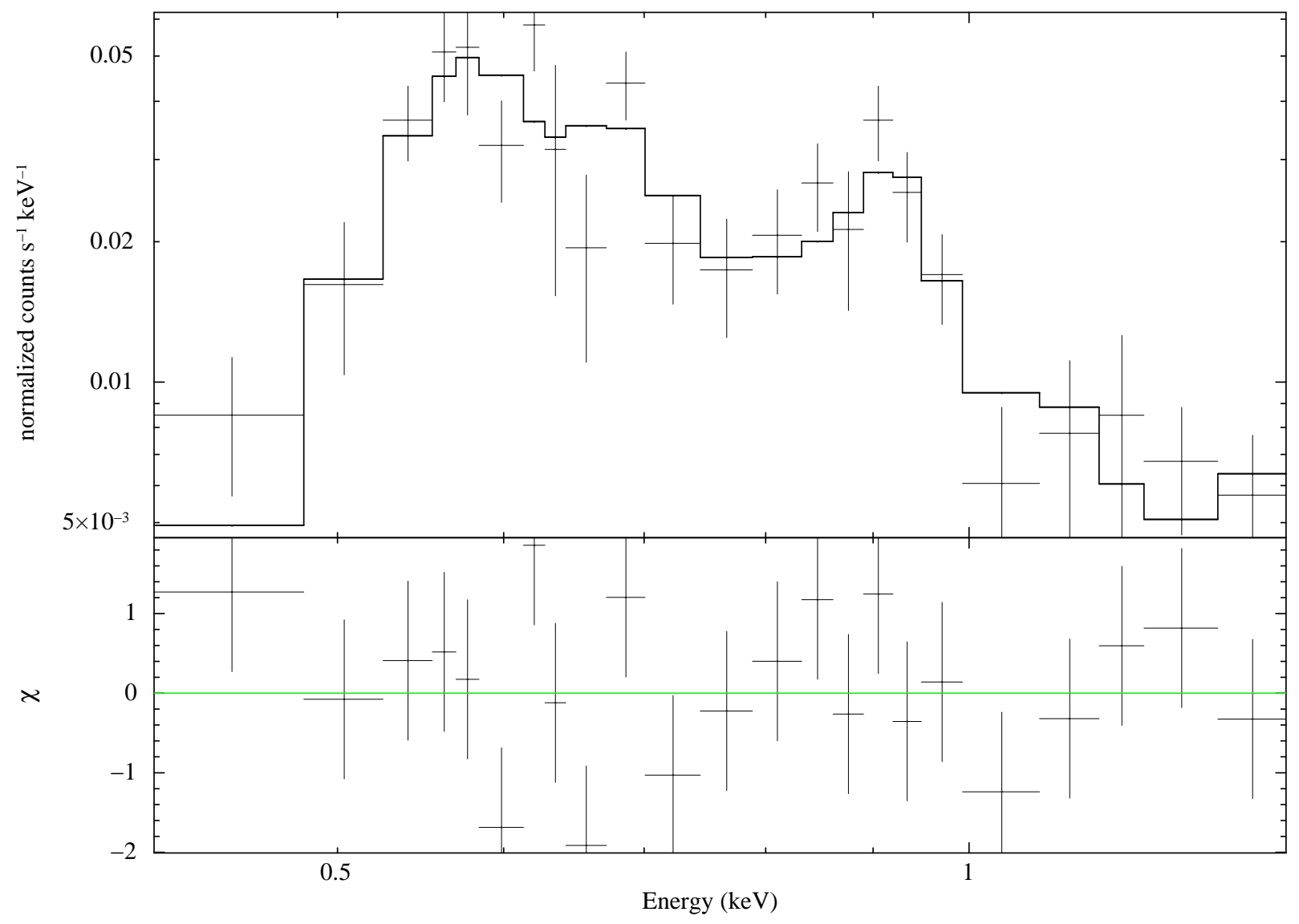

Fig. 5.- Chandra spectrum extracted from the diffuse emission region with the best fit thermal model overlaid. 


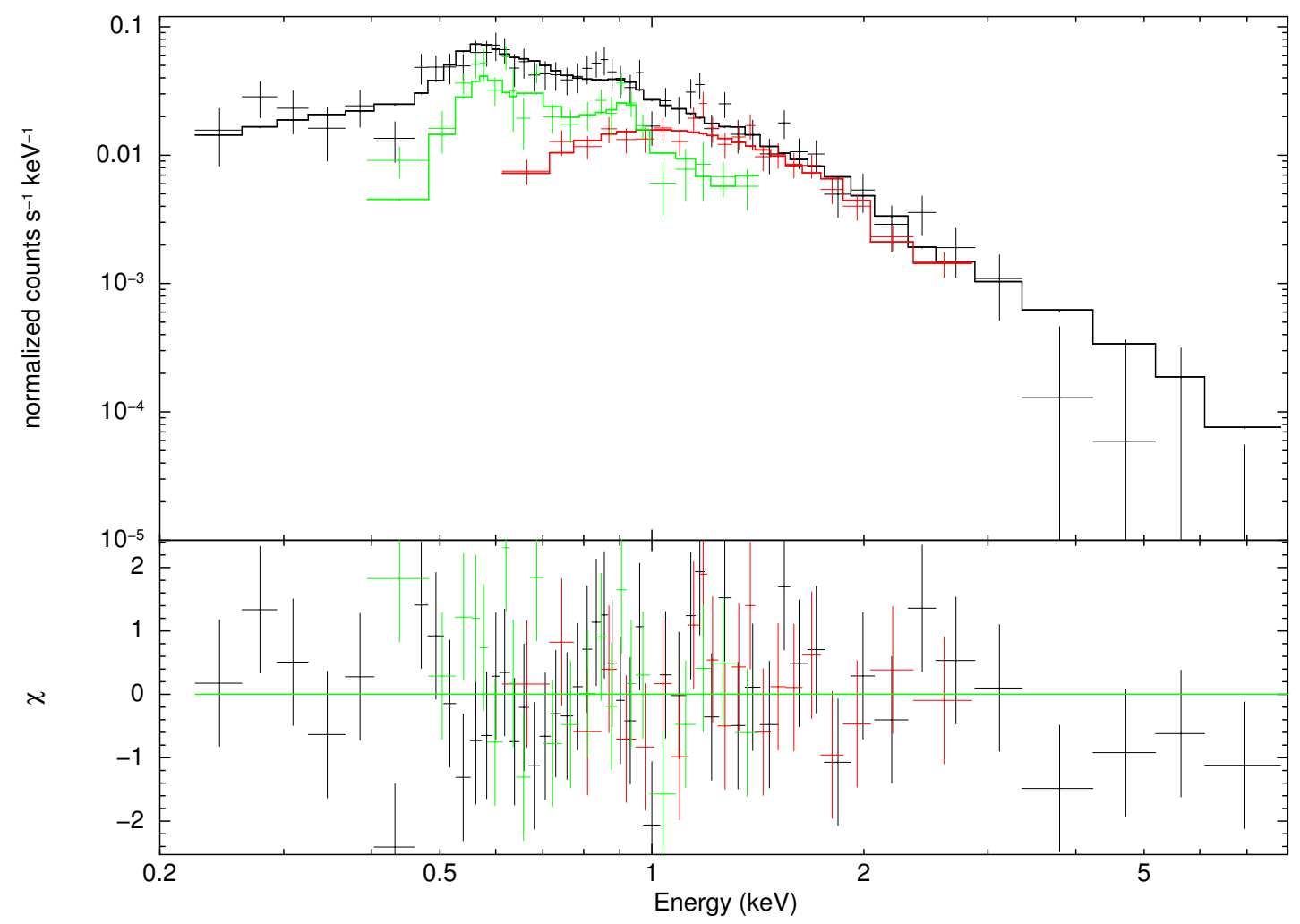

Fig. 6. - Co-fit Chandra (point source - red, diffuse emission - green) and XMM-Newton (black) spectra with best fit model overlaid. 


\section{is an unrelated background source.}

The powerlaw fit was reasonable even though we allowed the normalization to vary between the XMM-Newton and Chandra data. We did not detect significant flux variability. The best fit norm for the XMM-Newton data is higher than that for the Chandra data but just consistent with one another at the limits of the $90 \%$ confidence interval. Higher significance data are required to properly constrain the flux over multiple epochs to determine if flux variability is present.

The high temperature, nonequilibrium conditions in the diffuse region suggest that this gas has been recently shocked and point toward a younger SNR. Calculations of shock velocity and density support this conclusion. Solving the Rankine-Hugoniot relations in the strong shock case for a monatomic plasma $(\gamma=5 / 3)$, gives the post-shock temperature as a function of shock velocity, $k T=(3 / 16) \mu m_{p} v^{2}$. We assume that the plasma is fully ionized, so $\mu=0.6$ and $n_{e}=1.2 n_{H}$ This equation makes the implicit assumption that $T_{e} \sim T_{i o n}$. This is not necessarily the case for shock-heated plasmas (Ghavamian et al.2007), so our calculated velocities may be taken as a lower bound. The calculated velocity at the best fit temperature is $\sim 1100 \mathrm{~km} / \mathrm{sec}$, significantly decelerated from the initial blast wave velocity $(>5000 \mathrm{~km} / \mathrm{s})$, but still very fast. It does not appear that the diffuse emission is from particularly dense material even though it is probably dominated by shock-heated ISM as suggested by the SMC ISM abundances in the fit. The density is found using the norm parameter for the diffuse component, where norm $=\left[10^{-14} /\left(4 \pi D^{2}\right)\right] \int n_{e} n_{H} d V$. The integral contains the emission measure for the plasma which is dependent on the density and total emitting volume. A value of $60 \mathrm{kpc}$ is used for $D$ and we assume that $n_{e}=1.2 n_{H}$. We use the volume of a sphere with a radius equal to the average of the major and minor axes of the elliptical region used for extraction. We also include a filling factor, $f$, such that $V=4 / 3 \pi R^{3} f$. The resulting electron density is $0.09 / \sqrt{f} \mathrm{~cm}^{-3}$ supporting a low-density environment. Given that the filling factor is always less than 1 , and most likely for this remnant much less than 1 , this density is a lower limit and most likely larger. The low-density may explain the high temperature, nonequilibrium conditions and a conclusion of a young SNR may be premature. However, we can estimate the age of the remnant from the $\tau$ parameter since $\tau=n_{e} t$, where $t$ is this timescale. This calculation yields a shock time of $1800 \sqrt{f}$ years, thus supporting a lower age for this object. Finally, it is quite possible that this diffuse emission cannot be explained using a single set of plasma conditions. There is a need for higher signal-to-noise data to enable more detailed spatially resolved spectroscopy.

\section{Conclusions}

HFPK 334 is a young shell-type radio/X-Ray SNR with no optical or IR counterpart. The most striking feature of this SNR is the bright central object seen only in our X-ray observations. We argue that this central object with the best fit powerlaw $\Gamma=2.7 \pm 0.5$, could not be definitely associated with the remnant, as either a pulsar or compact central object. Therefore we propose that the central point source is a background object. The remnant itself appears as quite 
young, $<1800$ years, and our estimates of the remnant magnetic field $(\sim 90 \mu \mathrm{G})$ also favours younger age. The somewhat higher temperature and nonequilibrium conditions in the diffuse region suggest that this gas has been recently shocked. We report detections of scattered regions showing moderately high orders of polarisation at $20 \mathrm{~cm}$, with a peak value of $\sim 25 \pm 5 \%$, indicating the magnetic field is unordered.

The Australia Telescope Compact Array is part of the Australia Telescope National Facility which is funded by the Commonwealth of Australia for operation as a National Facility managed by CSIRO. The scientific results reported in this article are based on observations made by the Chandra $X$-ray Observatory (CXO). Based on observations obtained with XMM-Newton, an ESA science mission with instruments and contributions directly funded by ESA Member States and NASA. This research is supported by the Ministry of Education, Science and Technological Development of the Republic of Serbia through project No. 176005.

Facilities: ATCA, CXO, XMM-Newton.

\section{REFERENCES}

Arbutina, B., Urošević, D., Andjelić, M. M., Pavlović, M. Z., \& Vukotić, B. 2012, ApJ, 746, 79

Arnaud, K. A. 1996, in Astronomical Society of the Pacific Conference Series, Vol. 101, Astronomical Data Analysis Software and Systems V, ed. G. H. Jacoby \& J. Barnes, 17

Badenes, C., Borkowski, K. J., Hughes, J. P., Hwang, U., \& Bravo, E. 2006, ApJ, 645, 1373

Balucinska-Church, M., \& McCammon, D. 1992, ApJ, 400, 699

Bamba, A., Ueno, M., Nakajima, H., Mori, K., \& Koyama, K. 2006, A\&A, 450, 585

Berezhko, E. G., \& Völk, H. J. 2004, A\&A, 427, 525

Borkowski, K. J., Lyerly, W. J., \& Reynolds, S. P. 2001, ApJ, 548, 820

Borkowski, K. J., Sarazin, C. L., \& Blondin, J. M. 1994, ApJ, 429, 710

Bozzetto, L. M., Filipovic, M. D., Crawford, E. J., et al. 2010, Serbian Astronomical Journal, 181, 43

Bozzetto, L. M., Filipovic, M. D., Crawford, E. J., De Horta, A. Y., \& Stupar, M. 2012a, Serbian Astronomical Journal, 184, 69

Bozzetto, L. M., Filipovic, M. D., Crawford, E. J., et al. 2012b, Rev. Mexicana Astron. Astrofis., 48,41 
Bozzetto, L. M., Filipovic, M. D., Urosevic, D., \& Crawford, E. J. 2012c, Serbian Astronomical Journal, 185, 25

Bozzetto, L. M., Filipović, M. D., Urošević, D., Kothes, R., \& Crawford, E. J. 2014a, MNRAS, 440,3220

Bozzetto, L. M., Filipović, M. D., Crawford, E. J., et al. 2012d, MNRAS, 420, 2588

—. 2013, MNRAS, 432, 2177

Bozzetto, L. M., Kavanagh, P. J., Maggi, P., et al. 2014b, MNRAS, 439, 1110

Cajko, K. O., Crawford, E. J., \& Filipovic, M. D. 2009, Serbian Astronomical Journal, 179, 55

Chen, Y., Wang, Q. D., Gotthelf, E. V., et al. 2006, ApJ, 651, 237

Crawford, E. J., Filipovic, M. D., de Horta, A. Y., Stootman, F. H., \& Payne, J. L. 2008a, Serbian Astronomical Journal, 177, 61

Crawford, E. J., Filipović, M. D., Haberl, F., et al. 2010, A\&A, 518, A35

Crawford, E. J., Filipovic, M. D., \& Payne, J. L. 2008b, Serbian Astronomical Journal, 176, 59

De Horta, A. Y., Filipović, M. D., Bozzetto, L. M., et al. 2012, A\&A, 540, A25

De Horta, A. Y., Sommer, E. R., Filipović, M. D., et al. 2014, ArXiv e-prints

Filipović, M. D., Haberl, F., Winkler, P. F., et al. 2008, A\&A, 485, 63

Foster, A. R., Smith, R. K., Ji, L., \& Brickhouse, N. S. 2011, in 2010 NASA Laboratory Astrophysics Workshop, C2

Freeman, P., Doe, S., \& Siemiginowska, A. 2001, in Society of Photo-Optical Instrumentation Engineers (SPIE) Conference Series, Vol. 4477, Society of Photo-Optical Instrumentation Engineers (SPIE) Conference Series, ed. J.-L. Starck \& F. D. Murtagh, 76-87

Freeman, P. E., Kashyap, V., Rosner, R., \& Lamb, D. Q. 2002, ApJS, 138, 185

Fruscione, A., McDowell, J. C., Allen, G. E., et al. 2006, in Society of Photo-Optical Instrumentation Engineers (SPIE) Conference Series, Vol. 6270, Society of Photo-Optical Instrumentation Engineers (SPIE) Conference Series

Ghavamian, P., Laming, J. M., \& Rakowski, C. E. 2007, ApJ, 654, L69

Gooch, R. 1996, in Astronomical Society of the Pacific Conference Series, Vol. 101, Astronomical Data Analysis Software and Systems V, ed. G. H. Jacoby \& J. Barnes, 80

Green, D. A. 2009a, Bulletin of the Astronomical Society of India, 37, 45 
$\begin{array}{cccccc}\text { 2009b, } & \text { A } & \text { Catalogue of } & \text { Galactic } & \text { Supernova } & \text { Remnants } \\ \text { (http://www.mrao.cam.ac.uk/surveys/snrs/) } & & & \end{array}$

Grondin, M.-H., Sasaki, M., Haberl, F., et al. 2012, A\&A, 539, A15

Haberl, F. 2004, Advances in Space Research, 33, 638

Haberl, F., Filipović, M. D., Pietsch, W., \& Kahabka, P. 2000, A\&AS, 142, 41

Haberl, F., \& Pietsch, W. 2008, A\&A, 484, 451

Haberl, F., Sturm, R., Filipović, M. D., Pietsch, W., \& Crawford, E. J. 2012a, A\&A, 537, L1

Haberl, F., Filipović, M. D., Bozzetto, L. M., et al. 2012b, A\&A, 543, A154

Haberl, F., Sturm, R., Ballet, J., et al. 2012c, A\&A, 545, A128

Hamilton, A. J. S., Sarazin, C. L., \& Chevalier, R. A. 1983, ApJS, 51, 115

Hilditch, R. W., Howarth, I. D., \& Harries, T. J. 2005, MNRAS, 357, 304

Ishibashi, W., \& Courvoisier, T. J.-L. 2010, A\&A, 512, A58

Johanson, A. K., \& Kerton, C. R. 2009, AJ, 138, 1615

Kahabka, P., Pietsch, W., Filipović , M. D., \& Haberl, F. 1999, A\&AS, 136, 81

Kavanagh, P. J., Sasaki, M., Points, S. D., et al. 2013, A\&A, 549, A99

Liedahl, D. A., Osterheld, A. L., \& Goldstein, W. H. 1995, ApJ, 438, L115

Maccacaro, T., Gioia, I. M., Wolter, A., Zamorani, G., \& Stocke, J. T. 1988, ApJ, 326, 680

Maggi, P., Haberl, F., Bozzetto, L. M., et al. 2012, A\&A, 546, A109

Mao, S. A., Gaensler, B. M., Stanimirović, S., et al. 2008, ApJ, 688, 1029

Mazzotta, P., Mazzitelli, G., Colafrancesco, S., \& Vittorio, N. 1998, A\&AS, 133, 403

McEntaffer, R. L., Brantseg, T., \& Presley, M. 2012, ApJ, 756, 17

Müller, E., Staveley-Smith, L., Zealey, W., \& Stanimirović, S. 2003, MNRAS, 339, 105

Owen, R. A., Filipović, M. D., Ballet, J., et al. 2011, A\&A, 530, A132

Payne, J. L., White, G. L., Filipović, M. D., \& Pannuti, T. G. 2007, MNRAS, 376, 1793

Petre, R., Hwang, U., Holt, S. S., Safi-Harb, S., \& Williams, R. M. 2007, ApJ, 662, 988

Reynolds, S. P. 1998, ApJ, 493, 375 
Reynolds, S. P., \& Keohane, J. W. 1999, ApJ, 525, 368

Russell, S. C., \& Dopita, M. A. 1992, ApJ, 384, 508

Sault, R. J., Teuben, P. J., \& Wright, M. C. H. 1995, in Astronomical Society of the Pacific Conference Series, Vol. 77, Astronomical Data Analysis Software and Systems IV, ed. R. A. Shaw, H. E. Payne, \& J. J. E. Hayes, 433

Sault, R. J., \& Wieringa, M. H. 1994, A\&AS, 108, 585

Smith, R. K., Brickhouse, N. S., Liedahl, D. A., \& Raymond, J. C. 2001, ApJ, 556, L91

Strüder, L., Briel, U., Dennerl, K., et al. 2001, A\&A, 365, L18

Sturm, R., Haberl, F., Pietsch, W., et al. 2013, A\&A, 558, A3

Telezhinsky, I., Dwarkadas, V. V., \& Pohl, M. 2012, Astroparticle Physics, 35, 300

Udalski, A., Szymanski, M., Kubiak, M., et al. 1998, Acta Astron., 48, 147

van der Heyden, K. J., Bleeker, J. A. M., \& Kaastra, J. S. 2004, A\&A, 421, 1031

Williams, R. M., Chu, Y.-H., Dickel, J. R., et al. 2005, ApJ, 628, 704

Wilms, J., Allen, A., \& McCray, R. 2000, ApJ, 542, 914 